\title{
PROMOÇÃO DA SAÚDE MENTAL DO IDOSO NA ATENÇÃO BÁSICA: AS CONTRIBUIÇÕES DA TERAPIA COMUNITÁRIA
}

\author{
Fábia Barbosa de Andradeㄹ, Maria de Oliveira Ferreira Filha², Maria Djair Dias ${ }^{3}$, Antonia Oliveira Silva , \\ Iris do Céu Clara Costa ${ }^{5}$, Édija Anália Rodrigues de Lima ${ }^{6}$, Cristina Kátya Torres Teixeira Mendes ${ }^{7}$
}

\footnotetext{
${ }^{1}$ Mestranda do Programa de Pós-Graduação em Enfermagem da Universidade Federal da Paraíba (UFPB). Enfermeira da Secretaria Municipal de Saúde de Vila Flor-RN. Paraíba, Brasil. E-mail: fabiabarbosabr@yahoo.com.br

${ }^{2}$ Doutora em Enfermagem. Professora do Programa de Pós Graduação em Enfermagem da UFPB. Paraíba, Brasil. E-mail: marfilha@yahoo.com.br

${ }^{3}$ Doutora em Enfermagem. Professora do Programa de Pós-Graduação em Enfermagem da UFPB. Paraíba, Brasil. E-mail: mariadjair@yahoo.com.br

${ }^{4}$ Doutora em Enfermagem. Professora do Programa de Pós-Graduação em Enfermagem da UFPB. Paraíba, Brasil. E-mail: alfaleda@hotmail.com

${ }^{5}$ Doutora em Odontologia. Professora do Programa de Pós Graduação em Ciências da Saúde da UFRN. Rio Grande do Norte, Brasil. E-mail: iris_odontoufrn@yahoo.com.br

${ }^{6}$ Mestranda do Programa de Pós-Graduação em Enfermagem da UFPB. Paraíba, Brasil. E-mail: edijamiga@yahoo.com.br.

${ }^{7}$ Mestranda do Programa de Pós-Graduação em Enfermagem da UFPB. Bolsista da REUNI. Paraíba, Brasil. E-mail: criskadantas@ ig.com.br
}

\begin{abstract}
RESUMO: Para a Política Nacional de Saúde da Pessoa Idosa há dois megagrupos populacionais. Representando o primeiro grupo, estão idosos considerados independentes e o segundo envolve idosos frágeis. Nessa realidade se encontra inserida a Terapia Comunitária como espaço de partilha e promoção da saúde mental do idoso. Este estudo teve como objetivo conhecer as contribuições da Terapia Comunitária na vida dos idosos. Trata-se de um estudo exploratório com uma abordagem qualitativa, realizado com 19 idosos, em um município do Rio Grande do Norte. Utilizou-se um roteiro de entrevista e um caderno de campo. Emergiram as categorias temáticas: sentimento de empoderamento, partilha de experiências, contribuições da terapia comunitária e mudança de comportamentos revelando que a Terapia Comunitária representa uma estratégia de enfrentamento de desafios vivenciados no cotidiano dos idosos que frequentam os encontros, possibilitando uma significativa melhoria da autoestima e construção da teia de solidariedade na comunidade.
\end{abstract}

DESCRITORES: Saúde mental. Idosos. Atenção primária à saúde. Enfermagem.

\section{PROMOTING ELDERLY MENTAL HEALTH IN BASIC CARE: COMMUNITY THERAPY CONTRIBUTIONS}

\begin{abstract}
To the Brazilian National Elderly Health Policy, there are two population megagroups. Representing the first group are the senior citizens considered to be independent, while the second involves fragile senior citizens. Community Therapy is found to be inserted in such a reality as a space to share and to promote elderly mental health. The objective of this study is to better understand the contributions of Community Therapy in the senior citizens' lives. This is an exploratory study with a qualitative approach carried out with 19 senior citizens in a municipal district of Rio Grande do Norte, Brazil, using a structured interview and field notebook to collect the data. The following thematic categories emerged: sense of empowerment, sharing experiences, contributions of community therapy, and behavioral changes, revealing that Community Therapy represents a strategy which faces challenges lived in the dayto-day lives of the senior citizens who frequent the encounters. This makes a significant improvement of the community's self-esteem and the construction of a network of solidarity possible in the community.
\end{abstract}

DESCRIPTORS: Health mental. Aged. Primary health care. Nursing.

\section{LA PROMOCIÓN DE LA SALUD MENTAL DE LOS ANCIANOS EN LA ATENCIÓN BÁSICA: LAS CONTRIBUCIONES DE LA TERAPIA COMUNITARIA}

RESUMEN: La Política Nacional de Salud para los Ancianos incluye dos grandes grupos poblacionales: el primero, representado por los ancianos que son considerados independientes, y el segundo por los ancianos frágiles. Es en esa realidad en la que se inserta la Terapia Comunitaria como un espacio para compartir y promover la salud mental de los ancianos. El objetivo del estudio fue conocer las contribuciones de la Terapia Comunitaria en la vida de los ancianos. Se trata de un estudio exploratorio con abordaje cualitativo, realizado con 19 ancianos, en un municipio de Río Grande do Norte. Se utilizó un guión para la realización de las entrevistas, y un cuaderno de campo. Del análisis surgieron las siguientes categorías temáticas: la sensación de empoderamiento, el intercambio de experiencias, las contribuciones de la terapia comunitaria y el cambio de comportamiento, que revelan que la Terapia Comunitaria representa una estrategia para afrontar los retos que diariamente viven los ancianos que asisten a las reuniones, lo que les permite una mejora significativa en la autoestima y la construcción de una red de solidaridad en la comunidad.

DESCRIPTORES: Salud mental. Anciano. Atención primaria de salud. Enfermería. 


\section{INTRODUÇÃO}

Para a saúde coletiva, o envelhecimento populacional se apresenta como um dos maiores desafios, mormente nos países em desenvolvimento, onde a pobreza e a desigualdade social ganham destaque. Contudo, é importante ressaltar que essa transição demográfica reflete ganhos para o Estado e para a sociedade, pois esse envelhecimento da população é produto da redução da fecundidade, da mortalidade infantil e da mortalidade nas idades mais avançadas. ${ }^{1}$

Sabe-se que a evolução das ciências médicas efetivou importante contribuição para a longevidade populacional. Entretanto, verifica-se que essa condição demográfica tem interface com a progressão da qualidade de vida que, mesmo estando distante do ideal, encontra-se bem melhor do que à seis décadas atrás. Isso se revela por meio das melhorias na condição sanitária de modo geral, no trabalho e nas condições de moradia. No território brasileiro, bem como em outros países da América, essas melhorias repercutiram demograficamente na redução das taxas de mortalidade, especificamente nos primeiros anos de vida. ${ }^{2}$

O envelhecimento deve ser percebido com a naturalidade que lhe é intrínseca. E as pessoas envelhecidas deverão aprender a conviver positivamente com as limitações adquiridas, mantendo-se em atividade até alcançar idades mais avançadas. Esse envelhecimento deve ser compreendido como um processo, e a pessoa idosa como um indivíduo. Para a Política Nacional de Saúde da Pessoa Idosa há dois megagrupos populacionais de idosos, compreendidos por aqueles tidos como independentes e pelos considerados frágeis ou em processo de fragilização. Os primeiros envolvem os indivíduos idosos que apesar de serem acometidos por qualquer doença, conseguem se manter em atividade, seja no espaço familiar ou social. Já os últimos, correspondem aos idosos que por qualquer motivo manifestem alguma condição de fragilidade identificada e definida como tal pelo profissional de saúde que o avaliou. ${ }^{3}$

Ao alcançar a terceira idade, alguns indivíduos podem apresentar quadros psiquiátricos que chegam a ser comuns nessa faixa etária. Tais prejuízos mentais, de modo geral, incluem a demência, estados depressivos ou quadros psicóticos que são iniciados tardiamente. Contudo, há casos em que o transtorno teve início na juventude e o indivíduo alcançou a terceira idade, como por exemplo, a esquizofrenia, o transtorno afetivo bipolar, a distimia e transtornos ansiosos.
Logo, qualquer das situações referidas acarreta prejuízo sob a ótica funcional, assim como para a qualidade de vida. Vale salientar que parte considerável desses transtornos apresenta significativa melhora através de tratamento medicamentoso, somente, ou em conjunto com outras formas de terapia. ${ }^{4}$

A depressão é uma das doenças que atingem frequentemente os idosos, potencializando a probabilidade do desenvolvimento de incapacidade funcional. ${ }^{5}$ É uma patologia que demanda atenção, particularmente quando se dá pela primeira vez na terceira idade. Além de que, há casos em que os estados depressivos não são percebidos e, consequentemente, não tratados. E isso, compromete a saúde do idoso em intensidade relevante, a ponto de acarretar aumento da mortalidade por essa causa nessa faixa etária. ${ }^{4}$

O uso de antidepressivos deverá ser avaliado cuidadosamente, uma vez que esses psicotrópicos podem provocar efeitos adversos que poderão prejudicar a adesão ao tratamento ou trazer riscos para o idoso. Além disso, como essa clientela geralmente é acometida por mais de uma doença crônica, inevitavelmente depara-se com vários tratamentos que revelam a polifarmacoterapia. E essa condição vem a interferir na farmacocinética dos antidepressivos, muitas vezes elevando os níveis plasmáticos dessas drogas. ${ }^{5}$

A depressão se destacou entre os diagnósticos médicos identificados e corresponde a um problema comum e preocupante entre os idosos, podendo passar despercebida pelo mesmo e por seus familiares ou até mesmo precipitar o óbito dessa população. Além de que, pode ser acompanhada por outros sintomas, como a falta de sono e de apetite, entre outros. Diante do exposto, a discussão estará focada nesse problema que vem comprometendo a saúde mental de muitos idosos brasileiros. E, por isso, demanda reflexões quanto à atenção à saúde destes usuários, com destaque para a atenção primária, oferecida e administrada pelos gestores municipais.

Nesse contexto, enfoca-se que as políticas públicas deverão ser efetivas. E, para aquelas relacionadas ao envelhecimento populacional, o aspecto da efetividade deverá impreterivelmente, envolver setores distintos, como a saúde, a economia, o mercado de trabalho, a seguridade social e a educação. Isso revela o caráter integral intrínseco à compreensão da efetividade. ${ }^{6}$ Segundo a política brasileira que trata da saúde do idoso, a perda da capacidade funcional é considerada como proble- 
ma primordial para esses indivíduos, sendo esse problema entendido como a perda das faculdades físicas e mentais imprescindíveis para a realização de atividades basilares e instrumentais de sua vida cotidiana. ${ }^{7}$ Logo, diante dessa importante modificação no perfil populacional do Brasil, expressa-se a necessidade de aperfeiçoar as políticas públicas existentes, para ampliar sua capacidade de assistir com qualidade a esses cidadãos.

No Pacto em Defesa da Vida há seis prioridades, porém três delas se destacam no universo que envolve a saúde da população com 60 anos ou mais. Tais prioridades compreendem a saúde do idoso, a promoção da saúde e o fortalecimento da Atenção Básica (AB). Nesse contexto, a Política Nacional de Saúde da Pessoa Idosa vem reafirmar que a atenção primária deverá ser o meio de inserção inicial do idoso nos serviços de saúde do Sistema Único de Saúde (SUS), e contar com a referência da rede de serviços especializados de saúde, envolvendo a média e alta complexidade. Já a Política Nacional de Promoção da Saúde, dispõe de estratégias de implementação que deverão nortear as ações planejadas pelos profissionais da $\mathrm{AB}$ com o intuito de melhor assistir os idosos, em particular. E a Política Nacional de AB informa, sobretudo, que as ações deverão ser desenvolvidas através de práticas gerenciais e sanitárias democráticas e participativas, por meio do trabalho em equipe. ${ }^{8}$

Entre as responsabilidades gerais do gestor municipal do SUS pode-se destacar a atenção integral à saúde dos munícipes, de modo que essa responsabilidade esteja embasada no paradigma de solidariedade com o Estado e com a União. Nesse contexto, os municípios deverão assegurar ações de saúde integrais desenvolvidas sob um caráter interdisciplinar, de modo a promover equidade nas ações de atenção à saúde, contemplando as diversidades individuais assim como de grupos populacionais, através do ajustamento entre a oferta de serviços de saúde e as demandas, partindo do princípio de justiça social e ainda ampliar as possibilidades de inserção de grupos em situações desiguais, considerando as diversidades de cada localidade. Cabe aos municípios também a organização do acesso a serviços de saúde que primem pela resolutividade e qualidade na atenção básica, de modo a favorecer "[...] o planejamento, a programação pactuada integrada da atenção à saúde de seu território, explicitando a responsabilidade, [...] desenhando a rede de atenção e promovendo a humanização do atendimento" .9:7
$\mathrm{Na} A B$, os índices de reconhecimento dos sinais de depressão são muito baixos, o que compromete o emprego do tratamento adequado. Diante dessa dificuldade propõe-se a utilização de um instrumento de avaliação da saúde mental chamado Avaliação Multidimensional Rápida da Pessoa Idosa, que apresenta uma sequência de itens a serem avaliados pelo profissional de saúde. Um dos itens diz respeito à avaliação breve do estado de humor, e permite identificar a hipótese de depressão que, estando presente, será melhor avaliada pela Escala de Depressão Geriátrica. Essa escala destina-se ao uso de qualquer profissional da $\mathrm{AB}$, podendo ainda ser usada por leigos ou até mesmo ser autoaplicada. Vale salientar que ambos os instrumentos de avaliação, ora mencionados, estão disponíveis em um dos Cadernos de AB intitulado de Envelhecimento e Saúde da Pessoa Idosa. ${ }^{10}$

Diante da realidade anteriormente explana$\mathrm{da}$, percebe-se que as fragilidades presentes na atenção à saúde dos idosos, hoje alvo das políticas públicas de saúde, devem ser impulsionadoras com objetivo de melhoria da qualidade de vida destes. Nesse sentido, compreende-se que há possibilidades de minimizar ou eliminar alguns problemas que comprometem a saúde dessa clientela por meio de ações que potencializem a participação dos usuários no restabelecimento da sua saúde, através da prática da Terapia Comunitária (TC) enquanto estratégia de promoção da saúde mental em idosos na $A B$, funcionando como mola propulsora no atendimento de suas necessidades emocionais.

Desse modo, o tema abordado constitui-se como relevante, visto que está baseado na discussão e realização de um trabalho de saúde mental preventiva, em que todos os elementos culturais e sociais da comunidade se voltam para dar ênfase ao trabalho em grupo e à criação gradual da consciência social, suscitando ações terapêuticas transformadoras, apoiadas nas competências e na formação de uma teia de apoio, de modo que o presente estudo teve o objetivo de conhecer as contribuições da TC para a vida dos idosos.

\section{REFERENCIAL METODOLÓGICO}

Este é um estudo do tipo exploratório, com abordagem qualitativa, e foi realizado com 19 idosos, no período de março a junho de 2008, no município de Vila Flor, no Estado do Rio Grande do Norte, assistidos numa Unidade de Saúde da Família (USF). 
A USF possui uma área de abrangência com $100 \%$ de cobertura, perfazendo o equivalente a 2.666 habitantes no município de Vila Flor que são atendidos na AB. Destes habitantes, 1.342 são do sexo feminino e 1.324 do sexo masculino. ${ }^{11}$

O presente estudo insere-se no projeto Práticas de cuidado na rede formal e informal de saúde e sua execução iniciou-se em 2006 em municípios do Estado da Paraíba e do Rio Grande do Norte. Nesse ínterim, o estudo teve como recorte o município de Vila Flor-RN.

Para coleta de dados foram realizadas entrevistas e o caderno de campo, aliados à técnica observacional das pesquisadoras, para exploração das anotações no decorrer da discussão dos problemas elencados, além das estratégias de enfretamento diante do sofrimento mental dos idosos. A obtenção dos dados junto aos participantes do estudo deu-se por ocasião das reuniões de TC, semanalmente, no Clube Municipal de Vila Flor, em um total de seis encontros, contando com a participação média de 22 (63\%) usuários idosos.

Vale salientar que a promoção da saúde realizada no âmbito municipal lançou mão das atividades de TC, respeitando-se os artigos legais da Resolução No 196/96 do Conselho Nacional de Saúde, bem como foi realizado a assinatura do Termo de Consentimento Livre e Esclarecido pelos sujeitos que aceitaram participar do estudo. O Projeto foi aprovado pelo Comitê de Ética em Pesquisa do Centro de Ciências da Saúde da Universidade Federal da Paraíba, sob protocolo $\mathrm{N}^{\circ} 0059 / 2008$.

Para exploração do material empírico, foi utilizada a Análise Temática, ${ }^{12}$ cujo enfoque considera-se o texto como um objeto integralmente linguístico e histórico que, ao ser explorado, fez emergir categorias empíricas de análise, com seus respectivos temas, que serviram de referência para o processo inferencial e na interpretação dos resultados no referencial teórico da TC.

\section{RESULTADOS E DISCUSSÃO}

\section{Contribuições da terapia comunitária para idosos}

A TC foi instituída no município pesquisado na diretriz operacional do Pacto pela Vida, correspondendo a estratégica da Saúde Mental e em conformidade com a diretriz operacional que versa sobre a Política de Atenção à Saúde do Idoso, conforme a Portaria No 399/06, em que as ações implementadas devem ser norteadas, no âmbito municipal, através das ações de TC, a fim de promover a melhora da qualidade de vida dos idosos.

Após o surgimento das diretrizes operacionais do Pacto pela Saúde, o município de Vila Flor-RN, em cumprimento ao preconizado no elenco de promoção da saúde do idoso no Pacto pela Vida, em sua área estratégica da saúde mental, vem realizando encontros de TC com os idosos, por meio do trabalho de duas terapeutas comunitárias. A TC, que vem sendo desenvolvida há mais de 19 anos no Ceará, vem proporcionando aos munícipes de Vila Flor o aprendizado da partilha na comunidade, bem como o desenvolvimento de estratégias de promoção da Saúde Mental e de prevenção de transtornos mentais, sendo caracterizada como uma nova estratégia de integralidade, que se baseia na troca de experiência e vivências da comunidade a fim de nutrir a autonomia dos participantes.

A TC é composta pelas seguintes fases: 1) acolhimento, 2) escolha do tema, 3) contextualização, 4) problematização e 5) encerramento, em que a última ocorre com rituais de agregação. ${ }^{13}$

Nos encontros de TC em Vila Flor, vários temas são trabalhados e vêm fortalecendo a formação dos vínculos entre as pessoas. A terapia tem servido, ainda, como instrumento de reflexão sobre a realidade da população atendida no PSF e para facilitar o trabalho dos profissionais da equipe de saúde da família no sentido de melhorar o relacionamento com a comunidade no entendimento de suas necessidades. Das rodas de TC participam pessoas de faixas etárias diferentes, tanto homens como mulheres, podendo esses recuperarem sua autoestima e, através do processo de resiliência, empoderarem-se, tornando-se terapeutas de si mesmos, a partir da escuta de vida. Essa iniciativa vem promovendo em Vila Flor ações positivas no âmbito da saúde mental, traduzidas em aspectos de empoderamento e, por sua vez, em melhoria de qualidade de vida para todos.

Essa ação de promoção da saúde, intitulada TC já vem ocorrendo em vários estados brasileiros, além de ser experienciada na Franca, Suíça, Uruguai, Argentina e Venezuela. Atualmente, a TC expande-se no Brasil como espaço de ajuda mútua no âmbito comunitário, através de aproximadamente 38 pólos formadores. Conta-se, no momento, com a formação de cerca de 12.500 terapeutas comunitários. ${ }^{14}$ 
Dos resultados apreendidos a partir das falas dos idosos verifica-se a categoria - sentimento de empoderamento, que emergiu após a partilha dos problemas.

Aprendi aqui nos encontros de Terapia Comunitária, a ter mais confiança em Deus... (Rosa).

Percebe-se nesta fala que os encontros de TC resgataram a espiritualidade como elemento fortalecedor composto na identidade do idoso, uma vez que se acredita que seja na terceira idade que exista a maior aproximação com o Ser Supremo-Deus, como um sustentáculo nos momentos de sofrimento.

A escuta das histórias de vida de cada pessoa concorre para que todos se tornem co-responsáveis pela superação dos desafios do dia-a-dia, despertando para a solidariedade, a partilha, valorizando-se a dinâmica interna de cada ser humano, bem como sua capacidade de transformação social e coletiva, promovendo uma cultura de paz, podendo esta ser pautada na experiência da espiritualização. ${ }^{15}$

Para que essa partilha seja possível é proposto que na vivência grupal circulem os atributos das pessoas resilientes: 1 ) alta capacidade de resistência; 2) facilidade de construção coletiva; 3) alta capacidade para amar; 4) alta capacidade para crescer profissionalmente e 5) alta capacidade de troca com o mundo. ${ }^{16}$ Tais atributos são expressos nos exemplos da categoria - partilha de experiências.

Agradeço a vocês por essa terapia com idosos [...] a angústia que eu trazia ficou para trás e agora só tenho alegria e amor com todos aqui [...] (Vânia).

O foco da Terapia Comunitária Sistêmica não se encontra sobre os diagnósticos ou definições de problemas e teorias de mudança, mas sobre o sofrimento humano em qualquer uma de suas formas e manifestações, visando ações básicas de saúde comunitária, tendo como metas a prevenção, a mediação de crises e a inserção social, com vistas a reforçar os vínculos entre as pessoas da comunidade, mobilizar e valorizar as competências vindas da experiência, do saber local e da cultura. ${ }^{13}$

A fala da idosa Vânia revela a manifestação do sentimento de alegria e gratidão pela oportunidade de ser ouvido a ter o seu problema partilhado com outras pessoas, funcionando como redutor de seu sofrimento emocional, visto que quando a boca fala os órgãos saram.

É sabido que o tratamento da depressão no idoso deve primar primeiramente pela promoção da saúde e reabilitação psicossocial, e ainda prevenir possíveis recorrências, agravamento de outras patologias presentes e até mesmo o suicídio. Além disso, deverá melhorar a condição cognitiva e funcional do idoso e auxiliá-lo no enfrentamento das dificuldades. De tal modo que, para melhor tratar o idoso, deve-se fazer uso de algumas opções terapêuticas, como o atendimento individual, em que são prestadas orientações e acompanhamento, psicoterapia e farmacoterapia; atendimento em grupo; atividades comunitárias e atendimento à família. ${ }^{10}$

A TC caracteriza-se como uma estratégia de valorizar e respeitar as vivências de cada idoso na construção de saberes (científico/popular), desenvolvendo ações terapêuticas que proporcionam o equilíbrio físico e mental nos usuários da $A B$ fortalecendo assim sua identidade e cidadania. Nesse sentido, a AB, por meio do PSF, pode utilizar esse recurso como ferramenta em suas ações de saúde. ${ }^{17}$ Esses sentimentos encontram-se presentes na categoria - contribuições da terapia comunitária.

Estou saindo daqui aliviada por ter desabafado meus problemas ... me sinto agora fortalecida para dar valor a tudo aquilo que hoje vejo que faz parte de mim... (Margarida).

Essa fala expressa o resgate da autonomia do idoso após o encontro de TC, visto que seus valores passam a ganhar significância a partir do momento em que sua realidade é apresentada, partilhada e solidariamente compreendida.

Para não adoecer, precisamos verbalizar os sentimentos e as emoções que são escondidos, reprimidos; precisamos desabafar, confidenciar, partilhar nossa intimidade, nossos segredos, nossos pecados. O diálogo, a fala, a palavra, é um poderoso remédio uma excelente terapia. ${ }^{18}$

Os encontros de TC tecem redes de apoio e despertam possibilidades de mudanças, já que as pessoas da comunidade participam de uma mesma cultura e partilham entre si recursos de comunicação e laços de identidade, apresentando afinidades em seus sofrimentos e a busca de soluções para os mesmos. ${ }^{19}$

A categoria - mudança de comportamentos expressa relatos de alterações de postura/ opiniões dos participantes.

Depois que encontrei a Terapia Comunitária minha vida mudou muito ... saio aliviada levando a coragem de mãe que agora tem forças para ajudar a minha família e as outras pessoas que também precisam de alguma palavra de conforto...(Maria).

Torna-se perceptível na fala citada, que os encontros de TC promoveram mudanças de vida, 
através dos frutos de fortalecimento familiar, gerando apoio solidário que se estende para o seio da comunidade.

A TC constitui-se de um espaço público aberto, de ajuda mútua, onde se aborda tanto o indivíduo na sua singularidade como no seu contexto social, familiar e cultural. Ela se baseia na troca de experiência e vivências da comunidade a fim de nutrir a autonomia dos participantes que, através da partilha de vida e de experiências de forma horizontal, tornam-se co-responsáveis pela busca de soluções, valorizando-se a dinâmica interna de cada indivíduo e sua capacidade de transformação individual e coletiva. ${ }^{14}$

Para que isso seja possível, faz-se necessário entrar na cultura relacional, isso significa abordar uma paisagem social de conflitos, munida da compreensão de que se faz necessário retecer a imagem frágil da sociabilidade, cujos fragmentos estão exibidos no universo unidimensional do psicológico. Desse modo, trabalhar a cultura apresenta-se como alternativa de uma situação em que os investimentos sociais se eximem e as alternativas políticas estão embaralhadas. ${ }^{20}$

No espaço psicológico e social, o vínculo representa a responsabilização das equipes multiprofissionais pelo problema do indivíduo ou da coletividade, constituindo referências entre sujeitos, onde encontra no campo da $\mathrm{AB}$, a confluência de múltiplos saberes e práticas, conjugadas no esforço para identificar e atender às necessidades de saúde da população. ${ }^{21}$

A categoria - mudança de comportamentos, ainda pode ser realçada na seguinte fala: esses momentos de TC vem me ajudando a tomar decisões importantes na minha vida, com meus vizinhos e amigos...(Lucia).

No fragmento citado, se verifica que a TC auxiliou na tomada de decisões, direcionando a concretização resolutiva de situações de vida no meio familiar e com os demais da comunidade, visando a construção do complexus que envolve a rede solidária.

A institucionalização da rede diz respeito a um padrão de interdependência em que os atores envolvidos no processo descrevem ações coletivamente planejadas e deliberadamente orientadas em busca dos objetivos comuns. Essa constituição da rede resulta desse potencial de transformação social, cujos atores e organizações envolvidas podem promover mudanças nos padrões de identidade humana. ${ }^{20}$

Em estudo realizado com idosos na TC revelou que a estratégia mais utilizada por estes no enfrentamento das dificuldades cotidianas está o fortalecimento da espiritualidade em 57 citações $(24,56 \%)$, na qual segundo relatos dos participantes, é na oração e no cultivo a sentimentos de esperança movidos pelo desejo de conquista, que eles encontram conforto, estando a fé ganhando cada vez mais credibilidade no conceito da biomedicina, pois vários foram os momentos em que se percebeu o poder dela na cura. ${ }^{22}$

$\mathrm{Na}$ TC reverenciam-se todas as possibilidades de cura e soluções, buscando sempre o fortalecimento de sentimentos propulsores da vida como esperança, determinação, fé, fazendo com que cada um se conscientize que é responsável por uma parte da solução de seus problemas.

Esses resultados poderão ser mais positivos se ocorrer em um grupo disposto a se autoapoiar, em que a fala é seguida de imediato pelo feedback que advém da experiência de vida. Assim, são os encontros de TC um espaço de escuta que tem se mostrado eficaz na busca das melhores soluções para cada pessoa inserida nessa construção de saberes e práticas no cenário do cuidado. Essa terapia constitui uma estratégia eficaz porque atua nas questões que afligem o cotidiano, levando a construir um sentimento de pertença, uma melhoria das relações interpessoais, de autoestima, da saúde, da expressão emocional e da qualidade de vida das pessoas. ${ }^{18}$

Os idosos que participam das sessões de TC descobrem como são capazes de provocar mudanças em suas vidas e na de seus semelhantes. Por meio desses encontros a saúde mental vem se fortalecendo, particularmente, nas comunidades menos assistidas de infraestrutura efavorecimentos sociais de modo geral. Diante disso, a TC desperta em seus integrantes o poder de desenvolverem ferramentas de enfretamento para dores psicológicas advindas do cotidiano de desigualdades, contrariando alguns valores preconizados pela cultura capitalista, permeada por disputas, em que os poderosos se fortalecem permanentemente, e os desfavorecidos cada vez mais são lançados à margem daquilo que é socialmente imposto. Desse modo, de posse dos aspectos acima abordados, é percebida a grande contribuição das ações implementadas em nível comunitário, pois vêm se revelando como caminho para construção da rede solidária.

\section{CONSIDERAÇÕES FINAIS}

Este estudo apontou algumas contribuições da TC na vida dos idosos participantes, demonstrando assim que a mesma vem se consolidando 
enquanto uma estratégia eficiente não só no tocante ao fortalecimento dos princípios constitucionais que precisam se fazer presente na $A B$, mas também, como ferramenta para o resgate da autoestima de idosos.

A partilha de vida desses idosos vem auxiliando na redução do sofrimento emocional e, por sua vez, concorre para recuperação de vínculos familiares e sociais, objetivando a formação de redes de apoio solidário, conforme versa o Ministério da Saúde no Pacto pela Saúde em sua vertente operacional - Pacto em Defesa da Vida.

Desse modo, verificou-se através dos relatos e contribuições acima, que a TC vem funcionando como uma estratégia não só de fortalecimento da $\mathrm{AB}$, mas como um espaço de partilha de situações de sofrimento que afetam a saúde mental, considerando que para não adoecer faz-se necessário verbalizar os sentimentos, as emoções escondidas ou reprimidas. Esses resultados valorizam os momentos de desabafo, confidências e partilha de intimidade, uma vez que o diálogo, expresso na fala, constitui um poderoso efeito terapêutico, concretizados por meio das rodas de TC.

\section{REFERÊNCIAS}

1. Costa MFL. Epidemiologia do envelhecimento na Brasil, In: Rouquayrol MZ, Almeida Filho N, organizadores. Epidemiologia e saúde. $6^{\mathrm{a}}$ ed. Rio de Janeiro (RJ): Medsi; 2003. p.499-513.

2. Fleck MP, Chachamovich E, Trentini C. Development of the WHOQOL-OLD Module in Brazil. Rev Saúde Pública. 2006 Out; 40(5):785-91.

3. Ministério da Saúde(BR). Manual de Saúde da caderneta de saúde da pessoa idosa [online]. 2007 [acesso 2008 Jun 30]. Disponível em: http:/ / www.sbggpr. org.br/artigos/Caderneta \%20do\%20Idoso \%20-\%20 Manual\%20de\%20Preenchimento\%20MS.pdf

4. Ruipérez I, Llorente P. Depressão e suicídio: frequência, importância, manifestações e suspeita, cuidados, vigilância e tratamentos especiais. In: Ruipérez I, Llorente P. Geriatria. Rio de Janeiro (RJ): Mc Graw-hill; 2002. p. 243-51.

5. Leite VMM, Carvalho EMF, Barreto KML, Falcão IV. Depressão e envelhecimento: estudo nos participantes do programa Universidade Aberta à Terceira Idade. Rev Bras Saúde Matern Infant [online]. 2006 Jan-Mar [acesso 2008 Mar 27]; 6(1):31-8. Disponível em: http:/ / www.scielo.br/ pdf/rbsmi/v6n1/a04v6n1.pdf

6. Forlenza OV. Antidepressivos tricíclicos no tratamento farmacológico dos transtornos depressivos em idosos. Rev Psiq Clin [online]. 1999; Jul-Ago [acesso 2008 Mar 27]; 26(4):3-9. Disponível em: http://www.hcnet.usp.br/ipq/revista/r264/ $\operatorname{artigo}(172) \cdot h t m$

7. Rodrigues RAP, Kusumota L, Marques S, Fabrício SCC, Cruz IR, Lange C. Política nacional de atenção ao idoso e a contribuição da enfermagem. Texto Contexto Enferm [online]. 2007 Jul-Set [acesso 2008 Abr 13]; 16(3):536-45. Disponível em: http:/ / www. scielo.br/pdf/tccc/v16n3/a21v16n3.pdf

8. Ministério da Saúde (BR). Política Nacional de Saúde da Pessoa Idosa. Portaria GM/MS N²528 de 19 de outubro de 20: aprova a Política Nacional de Saúde da Pessoa Idosa. Brasília (DF); 2006.

9. Ministério da Saúde (BR), Portaria No 399/GM de 22 de fevereiro de 2006: divulga o Pacto pela Saúde 2006 - Consolidação do SUS e aprova as Diretrizes Operacionais do Referido Pacto. Brasília (DF); 2006:7.

10. Ministério da Saúde (BR), Secretaria de Atenção a Saúde, Departamento de Atenção Básica. Envelhecimento e Saúde da Pessoa Idosa. Brasília (DF); 2006.

11. Secretaria Municipal de Saúde (RN). Departamento de Atenção Básica. Relatório do Sistema de Informação da Atenção Básica (SIAB). Vila Flor (PB): SMS; 2008.

12. Bardin L. Análise de conteúdo. Lisboa (PT): Edições 70, 1995.

13. Grandesso MA. Terapia Comunitária: uma prática pós-moderna crítica - Considerações teóricoepistemológicas. In: Grandesso M, Barreto MR. Terapia Comunitária: tecendo redes para a transformação social, saúde, educação e políticas públicas. São Paulo: Casa do Psicólogo; 2007. p. 181-9.

14. Barreto A. Terapia Comunitária passo a passo. Fortaleza (CE): Gráfica LCR, 2005.

15. Holanda VR. A contribuição da Terapia Comunitária para enfrentamento das inquietações das gestantes [dissertação]. João Pessoa (PB): Universidade Federal da Paraíba. Programa de Pós Graduação em Enfermagem, 2006.

16. Ferreira Filha MO, Dias MD, Andrade FB, Alves TT. Terapia comunitária: principais problemas e estratégias de enfrentamento de seus participantes. In: Grandesso M, Barreto MR (Orgs.). Terapia Comunitária: Tecendo redes para a transformação social, saúde, educação e políticas públicas. São Paulo: Casa do Psicólogo; 2007: 295-300.

17. Ferreira Filha MO. Terapia Comunitária: uma ação básica de saúde mental. Projeto de Extensão (PROBEX), UFPB/CCS/DESPP; 2006.

18. Malheiros E. O uso da fala como instrumento restaurador dos males psicossomáticos e melhora da qualidade de vida. In: Grandesso M, Barreto MR. Terapia Comunitária: Tecendo redes para a transformação social, saúde, educação e políticas públicas. São Paulo (SP): Casa do Psicologo; 2007. p.143-5. 
19. Ferreira Filha MO, Dias MD, Andrade FB, Alves TT. Terapia Comunitária: principais problemas e estratégias de enfrentamento de seus participantes. In: Grandesso M, Barreto MR. Terapia Comunitária: Tecendo redes para a transformação social, saúde, educação e políticas públicas. São Paulo (SP): Casa do Psicólogo; 2007. p. 295-300.

20. Castel R. As metamorfoses da questão social: Uma crônica do salário. Petrópolis (RJ): Vozes; 1998.

21. Pessanha RV, Cunha FTS. A aprendizagem-trabalho e as tecnologias de saúde na Estratégia Saúde da
Família. Texto Contexto Enferm [online]. 2009 AbrJun [acesso 2009 Jul 10]; 18(2):233-40. Disponível em: http://www.scielo.br/pdf/tce/v18n2/05.pdf

22. Rocha IA, Braga LAV, Tavares LM, Andrade FB, Ferreira Filha $\mathrm{MaO}$, Dias MD, et al. A terapia comunitária como um novo instrumento de cuidado para saúde mental do idoso. Rev Bras Enferm [online]. 2009 Set-Out [acesso $2009 \mathrm{dez} 16]$; 62(5):687-94. Disponível em: http:// http://www. scielo.br/pdf/reben/v62n5/06.pdf 